\title{
Relationships between Members of the Mycoplasma mycoides Cluster as Shown by DNA Probes and Sequence Analysis
}

\author{
T. K. TAYLOR, ${ }^{*}$ J. B. BASHIRUDDIN, $\dagger$ AND A. R. GOULD \\ Australian Animal Health Laboratory, Commonwealth Scientific and Industrial \\ Research Organisation, Ryrie Street, Geelong, Victoria 3220, Australia
}

\begin{abstract}
A gene probe, CAP-21, which demonstrated interrelationships between the members of the Mycoplasma mycoides cluster was developed. The probe easily differentiated mycoplasmas in this cluster by clear and predictable hybridization patterns in Southern blots and separated the cluster into four groups. Strains of $M$. mycoides subsp. mycoides which were capable of causing contagious bovine pleuropneumonia composed one group. Strains of $M$. mycoides subsp. mycoides which did not cause contagious bovine pleuropneumonia together with strains of $M$. mycoides subsp. capri composed the second group. Mycoplasma capricolum and the F38 mycoplasmas formed a third group, while the bovine group 7 mycoplasmas composed a separate, fourth group. Further support for the above grouping of the cluster was obtained when amplified DNA analogous to the probe from one representative strain of each of the cluster members was sequenced and these data were used to construct a phylogenic tree. Contagious caprine pleuropneumonia is recognized as an important disease, and the etiological agent of this disease is now known to be the F38 mycoplasma. The CAP-21 probe did not differentiate between $M$. capricolum and the closely related F38 mycoplasma. A second probe, F38-12, which was capable of distinguishing these two mycoplasmas was made.
\end{abstract}

There are six mycoplasmas that make up the group known as the Mycoplasma mycoides cluster. The mycoplasmas in this group are pathogens of cattle, sheep, and goats. $M$. mycoides subsp. mycoides small colony (SC) is primarily a pathogen of cattle and causes contagious bovine pleuropneumonia (CBPP). M. mycoides subsp. mycoides large colony (LC) is usually found in goats, causing septicemia, arthritis, and pneumonia. $M$. mycoides subsp. capri causes pneumonia and arthritis in goats, and Mycoplasma capricolum causes septicemia, arthritis, and mastitis in goats and sheep. Two other groups of unnamed mycoplasmas complete the cluster. These are mycoplasmas represented by the F38 mycoplasma, which is now recognized as the agent of contagious caprine pleuropneumonia (CCPP), and bovine group 7 mycoplasmas, which cause arthritis and mastitis in cattle (8).

A number of difficulties have been identified in the classification of this group of organisms. Infrasubspecific varieties of $M$. mycoides subsp. mycoides (SC and LC forms) have been recognized. The two types are serologically indistinguishable but are quite different in pathogenicity; the SC form causes CBPP in cattle, while the LC form does not. Cottew and Yeats (9) showed that the two types could be separated when they correlated differences in cultural characteristics with differences in heat stability and variation in proteolytic activities. The presence of two enzymes, $\alpha$-glucosidase and ornithine transcarbamylase, was demonstrated in LC types but not in SC types (17). The ability to produce mycoplasmaemia in mice has also been used to separate LC and SC types (18).

$M$. mycoides comprises two subspecies, $M$. mycoides subsp. mycoides and $M$. mycoides subsp. capri. They can be differentiated by serological techniques but not by biochemical tests, as the reaction of the LC type of $M$. mycoides

\footnotetext{
* Corresponding author.

$\dagger$ Present address: Venerology and Infectious Diseases Unit, Department of Microbiology, Monash Medical School, Prahran, Victoria 3181, Australia.
}

subsp. mycoides is identical to that of $M$. mycoides subsp. capri. Although the type strains of the two subspecies are serologically distinct, significant cross-reactivity can occur between the subspecies with some antisera (7). Establishing the correct taxonomic position of the LC type of $M$. mycoides subsp. mycoides relative to $M$. mycoides subsp. mycoides SC and $M$. mycoides subsp. capri has been made difficult because of conflicting results. DNA homology studies have shown that $M$. mycoides subsp. mycoides LC was more closely related to $M$. mycoides subsp. mycoides SC than to $M$. mycoides subsp. capri (2). Protein studies have indicated the opposite $(6,15,16)$.

There have also been problems in classifying the two unnamed mycoplasmas belonging to the cluster. Some field isolates of the F38 mycoplasma have been shown to crossreact with $M$. capricolum and bovine group 7 mycoplasma antisera (4). Also, DNA studies have shown an $80 \%$ relatedness between the type strains of the F38 mycoplasma and $M$. capricolum which indicated that the F38 mycoplasmas should become a subspecies of $M$. capricolum (5). Onedimensional (1D)- and 2D-polyacrylamide gel electrophoresis (PAGE) analyses of proteins also group these two mycoplasmas together $(6,16)$.

The bovine group 7 mycoplasmas have remained an unclassified group for many years. Very strong serological cross-reactions have been demonstrated between bovine group 7 and F38 mycoplasmas, and on this basis it was proposed that bovine group 7 mycoplasmas form a biovar of the F38 subspecies of $M$. capricolum (4). DNA homology studies, on the other hand, suggest that bovine group 7 mycoplasmas are equally related to the $M$. mycoides species and $M$. capricolum and that because of this, they should form a separate species (5).

Many different techniques have been used to try to clarify the relationships between these organisms. These techniques have shown a very complicated range of relationships with conflicting results, and as a result an ad hoc committee established by the Subcommittee on the Taxonomy of the Mollicutes has not been able to reach agreement on the classification of the members of the $M$. mycoides cluster but 
TABLE 1. List of the members of the $M$. mycoides cluster used in this study as well as their host species of origin and country of origin

\begin{tabular}{|c|c|c|c|}
\hline Mycoplasma & Strain & $\begin{array}{l}\text { Species } \\
\text { of origin }\end{array}$ & $\begin{array}{l}\text { Country } \\
\text { of origin }\end{array}$ \\
\hline \multirow{2}{*}{$\begin{array}{l}\text { M. mycoides subsp. } \\
\text { mycoides SC }\end{array}$} & Gladysdale & Bovine & Australia \\
\hline & PG1 & Bovine & Unknown \\
\hline \multirow{9}{*}{$\begin{array}{l}\text { M. mycoides subsp. } \\
\text { mycoides LC }\end{array}$} & Y Goat & Caprine & Australia \\
\hline & KH1 & Caprine & Australia \\
\hline & cov2 & Caprine & Turkey \\
\hline & LB2 & Caprine & Australia \\
\hline & 801 & Bovine & Australia \\
\hline & M243/67 & Caprine & United States \\
\hline & OSB42 & Caprine & Nigeria \\
\hline & EZG & Caprine & Nigeria \\
\hline & F30 & Caprine & Kenya \\
\hline \multirow{5}{*}{$\begin{array}{l}\text { M. mycoides subsp. } \\
\text { capri }\end{array}$} & PG3 & Caprine & Turkey \\
\hline & BQT & Caprine & Turkey \\
\hline & YC & Caprine & Australia \\
\hline & $\mathrm{ZZ}$ & Caprine & Australia \\
\hline & N108 & Caprine & Kenya \\
\hline \multirow[t]{2}{*}{ M. capricolum } & California Kid & Caprine & United States \\
\hline & 3220 & Caprine & Australia \\
\hline F38 mycoplasma & F38 & Caprine & Kenya \\
\hline \multirow{2}{*}{$\begin{array}{l}\text { Bovine group } 7 \text { myco- } \\
\text { plasma }\end{array}$} & $\mathrm{N} 29$ & Bovine & Australia \\
\hline & R2222 & Bovine & Australia \\
\hline
\end{tabular}

emphasized the need for further DNA studies. This paper describes the use of gene probes and nucleic acid sequencing to further differentiate and classify this group of mycoplasmas and provides the basis of a test for distinguishing the etiological agents of CBPP and CCPP.

\section{MATERIALS AND METHODS}

Organisms. Table 1 lists the mycoplasma species used in this study as well as their host species of origin and country of origin. Other organisms used were laboratory strains of Mycoplasma hyorhinis, Mycoplasma orale, Acholeplasma laidlawii, Escherichia coli, and Pasteurella multocida.

Cloning and sequencing of probes. Two probes were prepared. One probe, CAP-21, was used in the differentiation of members of the $M$. mycoides cluster. $M$. mycoides subsp. capri was digested with the restriction endonuclease EcoRV.

TABLE 2. Primers used in sequencing and PCR

\begin{tabular}{|c|c|c|c|}
\hline $\begin{array}{c}\text { Primer } \\
\text { no. }\end{array}$ & $\begin{array}{l}\text { Nucleo- } \\
\text { tide no. }\end{array}$ & Primer sequence $\left(5^{\prime} \rightarrow 3^{\prime}\right)^{b}$ & Direction \\
\hline 1 & 11 & TAGAGGTACTTTAGATACTCAAGG & Forward \\
\hline 2 & 207 & AAGAGATGTTTTAGCAGATCC & Forward \\
\hline 3 & 281 & GGTAAAAGAGGAATTGC & Forward \\
\hline 4 & 649 & TAATCAAATTAGAAAGG & Forward \\
\hline 5 & 837 & CTCAAGAGCAAGAACGT & Forward \\
\hline 6 & 1000 & CAATCTGGTGTTGAACC & Forward \\
\hline 7 & 1223 & GGTTTTCCG/ATCAAACTCATAAGC & Reverse \\
\hline 8 & 1422 & TTAATGCTGATTTCTTCCC & Forward \\
\hline 9 & 1509 & GATATCTAAAGGTGATGGT & Reverse \\
\hline 10 & $237^{c}$ & TTCTTCTGCTTTTTTAATTGC & Forward \\
\hline
\end{tabular}

${ }^{a}$ The numbers correspond to the nucleotide positions of the first base of the primer relative to the total sequence in Fig. 4A for CAP-21.

${ }^{b}$ Oligonucleotides were synthesized by using a DNA synthesizer (Applied Biosystems) according to manufacturer's instructions and purified by using the oligonucleotide purification cartridge columns supplied.

${ }^{c}$ For primer 10 the nucleotide number corresponds to the first base of primer relative to the total sequence in Fig. 5A for F38-12.
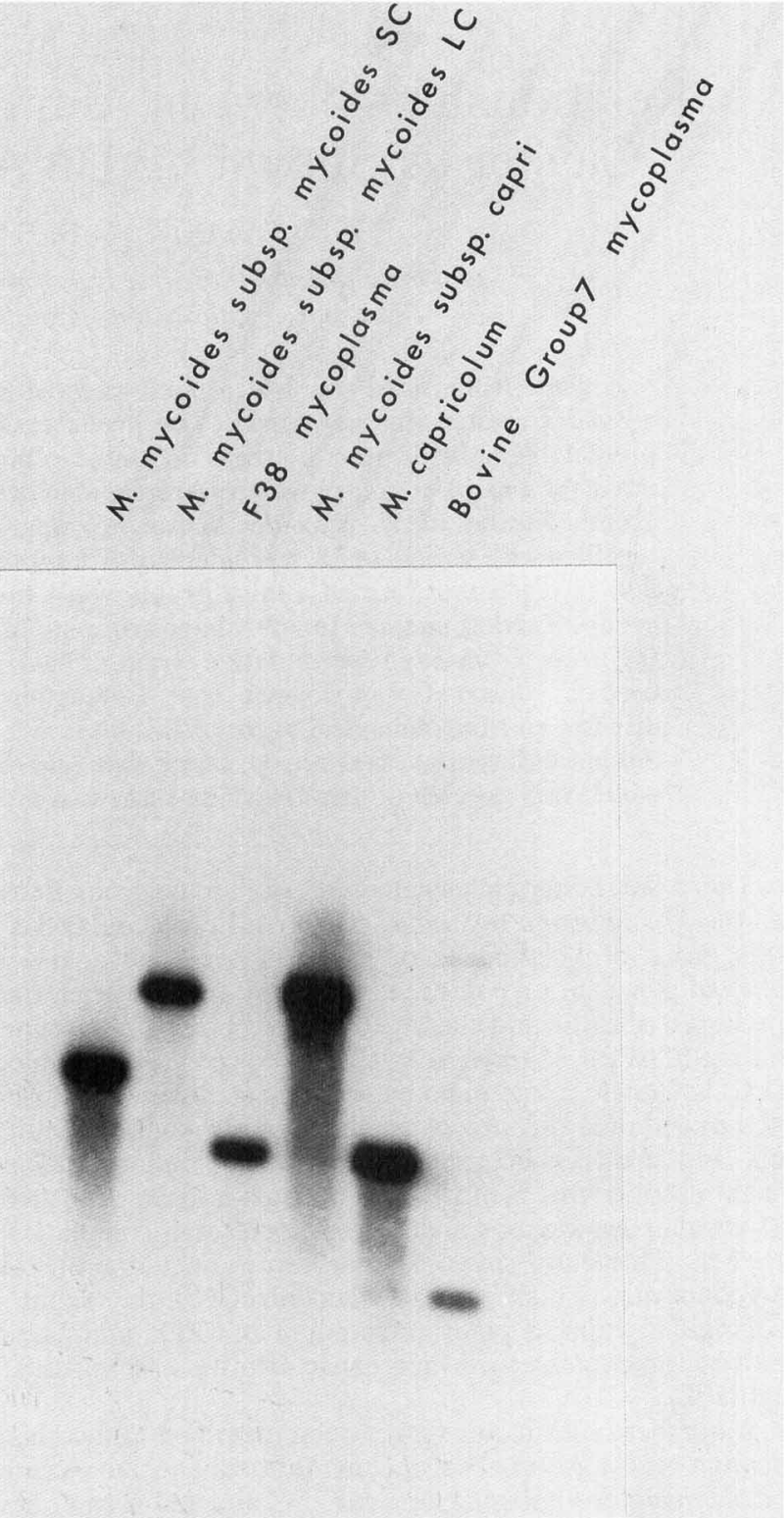

FIG. 1. Comparison of DNA from representative members of the $M$. mycoides cluster, $M$. mycoides subsp. mycoides SC (Gladysdale), M. mycoides subsp. mycoides LC (Y Goat), F38 mycoplasma (original isolate), $M$. mycoides subsp. capri (ZZ), M. capricolum (California Kid), and bovine group 7 mycoplasma (R2222), digested with TaqI and probed with ${ }^{32} \mathrm{P}$-labelled CAP-21. Hybridization bands can be seen with all organisms.

Several fragment size classes were inserted into $\mathrm{M} 13 \mathrm{mp} 8$ at the SmaI site. Clones produced were screened for the presence of an insert, and several clones with inserts of 500 to $2,000 \mathrm{bp}$ were chosen for further investigation. These were tested as probes by using the DNA of SC (Gladysdale) and LC (Y Goat) strains of $M$. mycoides subsp. mycoides by Southern hybridization. One clone CAP-21 was sequenced by using Sequenase version 2.0 (United States Biochemical Corp.) with primers 2, 4, 6, and 8 (Table 2) and M13 forward primer (supplied with kit).

The second probe, F38-12, was used to differentiate F38 mycoplasma and $M$. capricolum. Genomic DNA from F38 


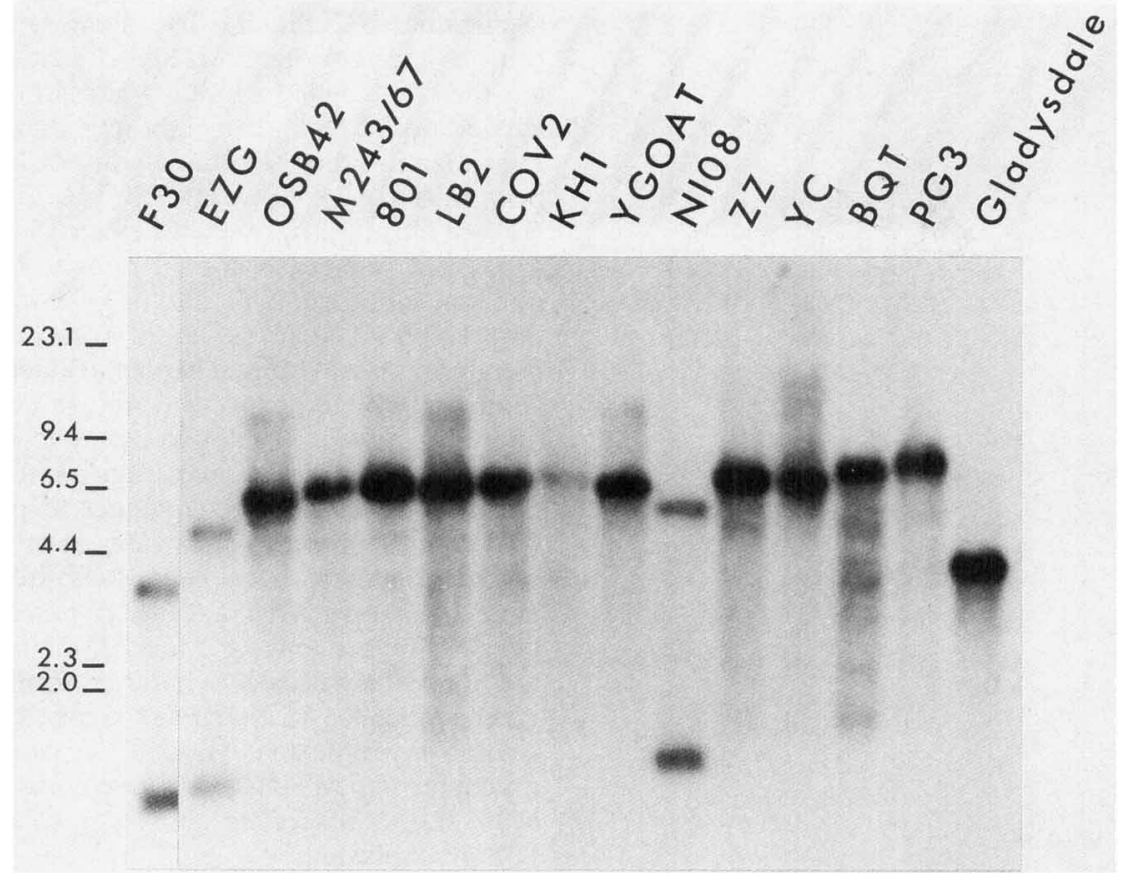

FIG. 2. Comparison of DNA from strains of $M$. mycoides subsp. mycoides LC and $M$. mycoides subsp. capri TaqI digested and probed with ${ }^{32}$ P-labelled CAP-21. LC strains are Y Goat, KH1, COV2, LB2, 801, M243/67, OSB42, EZG, and F30. PG3, BQT, YC, ZZ, and N108 are strains of $M$. mycoides subsp. capri. M. mycoides subsp. mycoides SC strain Gladysdale is used as a comparison. The relative positions of size markers are indicated; the sizes are in kilobase pairs. F30, EZG, and N108 deviate from the general hybridization pattern.

mycoplasma was digested with the restriction endonuclease EcoRI. Fragments were randomly inserted into M13mp18 at the EcoRI site. Clones produced were screened for the presence of an insert. Several clones were chosen for testing with F38 mycoplasma and $M$. capricolum (California Kid) by Southern hybridization. F38-12 was chosen and sequenced by using Sequenase version 2.0 (United States Biochemical Corp.) with primer 10 (Table 2) and M13 forward primer.

DNA extraction, PCR amplification, and sequencing. Representative members of the $M$. mycoides cluster selected were $M$. mycoides subsp. mycoides SC strain Gladysdale, $M$. mycoides subsp. mycoides LC strain Y Goat, $M$. mycoides subsp. capri ZZ, $M$. capricolum California Kid, F38 mycoplasma original isolate, and bovine group 7 mycoplasma strain R2222. Template DNA from these organisms was extracted after treatment with proteinase $\mathrm{K}$ by using phenol as described in reference 19 . Regions analogous to CAP-21 were amplified by polymerase chain reaction (PCR) using primers 1 and 9 (Table 2). Amplification was for 30 cycles in a total volume of $50 \mu \mathrm{l}$ of reaction mixture consisting of $10 \mathrm{mM}$ Tris- $\mathrm{HCl}$ (pH 8.3); $50 \mathrm{mM} \mathrm{KCl} ; 1.5 \mathrm{mM}$ $\mathrm{MgCl}_{2} ; 0.001 \%$ (wt/vol) gelatin; $200 \mu \mathrm{M}$ (each) dATP, dGTP, dTTP, and dCTP; and 1.5 U of Taq polymerase (PerkinElmer Cetus). Each primer was used at 50 pmol. Each amplification cycle consisted of annealing at $50^{\circ} \mathrm{C}$ for $1 \mathrm{~min}$, extension at $72^{\circ} \mathrm{C}$ for $2 \mathrm{~min}$, and denaturation at $94^{\circ} \mathrm{C}$ for 1 min. PCR product $(5 \mu \mathrm{l})$ was then electrophoresed on a $0.8 \%$ agarose gel, and the PCR product was visualized by staining with ethidium bromide. The DNA band was excised and purified by using Geneclean (Bio 101, La Jolla, Calif.) and resuspended in $5 \mu \mathrm{l}$ of $\mathrm{H}_{2} \mathrm{O}$. This was used to make singlestranded DNA by using asymmetric PCR and conditions as described above except that amplification was for 40 cycles and the primer concentrations were made so that one was in excess $(50 \mathrm{pmol})$ and one was in a limiting amount $(0.5$

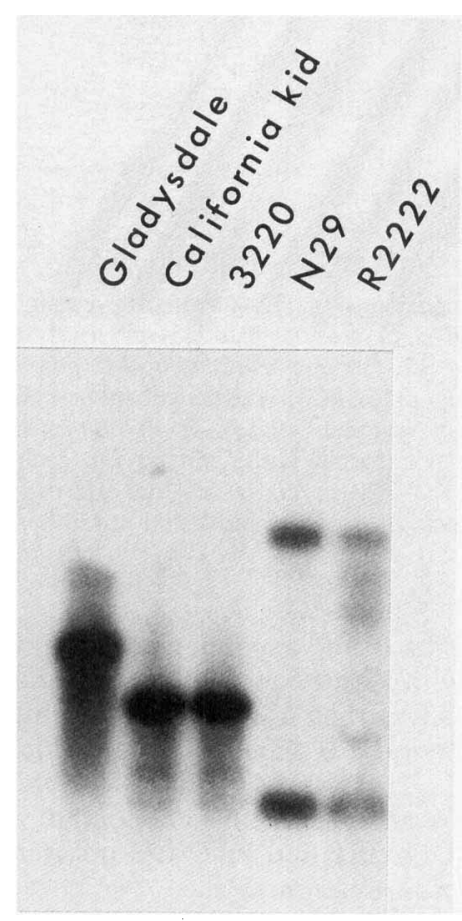

FIG. 3. Comparison of DNA from strains of $M$. capricolum (California Kid and 3220) and bovine group 7 mycoplasmas (N29 and R2222) TaqI-digested and probed with ${ }^{32}$ P-labelled CAP-21. $M$. mycoides subsp. mycoides SC strain Gladysdale is used as a reference. 


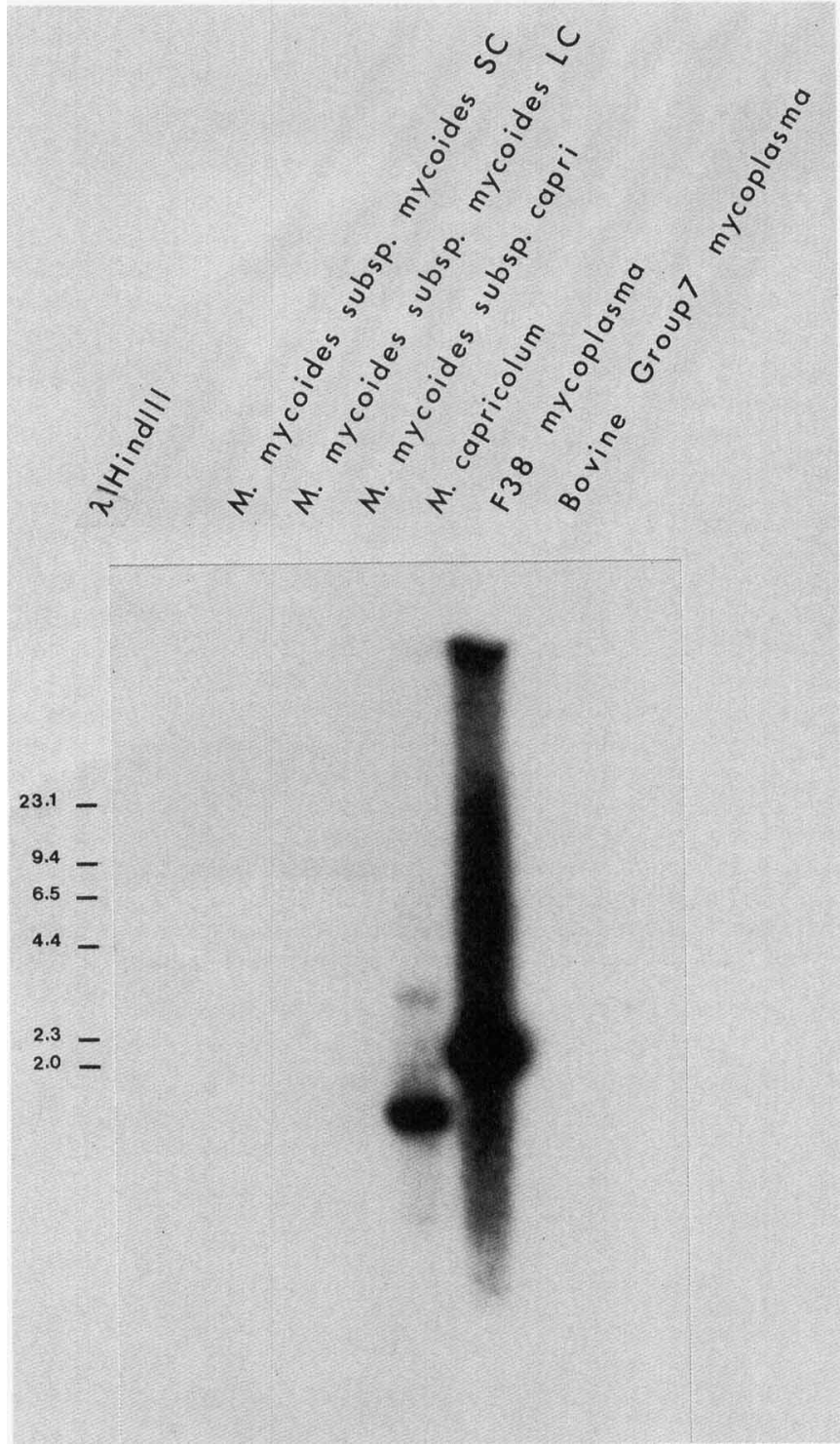

FIG. 4. Comparisons of DNA from representative members of the $M$. mycoides cluster digested with $R s a I$ and probed with ${ }^{32} \mathrm{P}$-labelled F38-12. Strains used were $M$. mycoides subsp. mycoides SC (Gladysdale), M. mycoides subsp. mycoides LC(Y Goat), F38 mycoplasma (original isolate), $M$. mycoides subsp. capri (ZZ), M. capricolum (California Kid), and bovine group 7 mycoplasma (R2222). Only the F38 mycoplasma and $M$. capricolum produce hybridization bands. Sizes are indicated in kilobase pairs.

pmol). The DNA in the asymmetric PCR product was then precipitated with isopropanol and resuspended in $7.5 \mu \mathrm{l}$ of $\mathrm{H}_{2} \mathrm{O}$ (14). This was then used in a sequencing reaction using Sequenase version 2.0 (United States Biochemical Corp.) and primers $1,2,3,4,5,7$, and 9 (Table 2). The DNA sequences were analyzed, and a dendogram was constructed by using the DNAIST and KITSCH programs of reference 10 as described in reference 12 .

Southern hybridization. Purified genomic DNA (5.0 to 10.0 $\mu \mathrm{g})$ was digested overnight with $50 \mathrm{U}$ of the restriction endonuclease TaqI (when CAP-21 probe was used) or RsaI (when F38-12 probe was used), electrophoresed in $0.8 \%$ agorose gels, and transferred to a nitrocellulose membrane
(Schleicher \& Schuell). The membrane was baked at $80^{\circ} \mathrm{C}$ prior to prehybridization. Hybridization was performed using the ${ }^{32} \mathrm{P}$-labelled probe at $5 \times 10^{5} \mathrm{cpm} / \mathrm{ml}$ (19) and was carried out at $65^{\circ} \mathrm{C}$ overnight in a shaking water bath. After hybridization the membrane was washed as follows: (i) two brief rinses in $2 \times \mathrm{SSC}(1 \times \mathrm{SSC}$ is $0.15 \mathrm{M} \mathrm{NaCl}$ plus $0.015 \mathrm{M}$ sodium citrate), (ii) two washes for $15 \mathrm{~min}$ each in $2 \times \mathrm{SSC}$ at $65^{\circ} \mathrm{C}$, (iii) one wash for $30 \mathrm{~min}$ in $2 \times \mathrm{SSC}-0.1 \%$ sodium dodecyl sulfate at $65^{\circ} \mathrm{C}$, and (iv) two washes for 15 min each in $0.1 \times$ SSC. Excess liquid was blotted away, and the membrane was wrapped in plastic and placed onto $\mathrm{X}$-ray film (Fuji AR100). Exposure was allowed for 4 to $16 \mathrm{~h}$ at $-80^{\circ} \mathrm{C}$, and the film was developed by standard methods.

Nucleotide sequence accession numbers. The sequence of the CAP-21 insert and sequences of regions homologous to CAP-21 in representative strains of other members of the $M$. mycoides cluster have been submitted to GenBank. They have been given the accession numbers which are listed below. The sequence for the F38-12 insert was also submitted, and the accession number for this sequence is also given: M96586, M. mycoides subsp. capri; M96587, bovine group 7 mycoplasma; M96588, $M$. capricolum; M96589, F38 mycoplasma; M96590, M. mycoides subsp. mycoides SC; M96591, M. mycoides subsp. mycoides LC; and M96592, F38 mycoplasma.

\section{RESULTS}

Southern hybridization. Southern hybridization with the CAP-21 probe was used to study the interrelationships of the $M$. mycoides cluster. This probe in combination with TaqI digestion resulted in a very simple hybridization pattern of one or two bands which facilitated the easy identification of the members of the $M$. mycoides cluster. The probe differentiated the $M$. mycoides cluster into groups and separated the two infrasubspecific types of $M$. mycoides subsp. mycoides (SC and LC forms), enabling organisms which are capable of causing CBPP to be distinguished from the others. The probe also differentiated the two subspecies of $M$. mycoides represented by PG1 and PG3. PG3, the type strain of $M$. mycoides subsp. capri, and Y Goat, the representative strain of $M$. mycoides subsp. mycoides LC, both gave similar hybridization patterns. The probe grouped $M$. capricolum and F38 mycoplasmas together, while the bovine group 7 mycoplasmas made a separate, distinct grouping (Fig. 1).

A total of five strains of $M$. mycoides subsp. capri and nine strains of $M$. mycoides subsp. mycoides LC were tested. Eleven of the 14 strains produced similar hybridization patterns. Among the three strains which showed different hybridization patterns were one strain of $M$. mycoides subsp. capri, N108, and two strains of $M$. mycoides subsp. mycoides LC, EZG and F30. Strains EZG and N108 appeared to be the same as each other and different from F30. All 3 differed from the other 11 strains in having an additional hybridization band and presumably contained an extra TaqI site (Fig. 2). The bovine group 7 mycoplasmas, strain R2222 and the representative strain $\mathrm{N} 29$, along with strains of $M$. capricolum, California $\mathrm{Kid}$ and 3220 , were tested with CAP-21 using the Gladysdale strain of $M$. mycoides subsp. mycoides SC as a comparison. The two strains of the bovine group 7 mycoplasmas hybridized in identical positions, as did the two strains of $M$. capricolum (Fig. 3). To differentiate M. capricolum from F38 mycoplasmas, a second probe, F38-12, was developed. Genomic DNAs of representative strains of each member of the $M$. mycoides cluster were 


\begin{tabular}{|c|c|c|c|c|c|c|}
\hline AT & TAGAGGTACT & TTAGATACTC & AAGGTGTAGC & AAAACGTTCT & CAAGGACGTT & 50 \\
\hline CACTATATGG & AGTAAAAAGA & CCAAAAGTTA & AAAAATAATA & AATCAAATTA & ATAAGTGTGT & 120 \\
\hline ATATGAATTA & AAAAGAACAC & ATTAAAAGTA & GCATATTTAT & ATTAAAAAGA & AAGGAGTTAT & 180 \\
\hline TACCATGCGT & AAAAATAGAG & CAGAAAAAAG & AGATGTTTTA & GCAGATCCAA & TTTATAATTC & 240 \\
\hline TAAATTAGTT & ACTCGTGCAA & TTAATAAAAT & TATGTTAGAT & GGTAAAAGAG & GAATTGCTCA & 300 \\
\hline GTCAATTATT & TATGATGCAT & TTAATATAAT & TAAAGAAAAA & ACTAATAAAG & AACCAATTGA & 360 \\
\hline AGTATTTAAT & AAAGCTATTG & AAAATATTAA & АССТСАСТTA & GAATTAAAAG & ICGTAT & 420 \\
\hline TGGAGGAGCT & AACTATCAAG & TTCCTGTAGA & AGTTTCAGCT & GAAAGACAAA & TCACTTTAGC & 480 \\
\hline TTTACGTTGA & TTAATTAATT & ATGCAAGATT & AAGAAACGAA & AAAGTTATGA & CAATTAAATT & 540 \\
\hline AGCTAATGAA & ATTATTGATC & GATCAAATAA & TATTGGTGGA & TCAGTTAAAA & AACGTGAAGA & 600 \\
\hline TACTCATAAA & ATGGCAGAAC & GAAATAAAGC & GTTTGCTCAT & TATCGTTGAT & AATCAAATTA & 660 \\
\hline GAAAGGAAAA & TACGAAATGG & CTAGAGAATA & CAGTTTATTA & AATACTCGTA & ATATTGGTAT & 720 \\
\hline TATGGCTCAT & ATTGATGCTG & GTAAGACTAC & TACTACTGAA & CGTATTTTAT & TTCATACTGG & 780 \\
\hline AAAAATTCAC & AAAATAGGTG & AAACACATGA & AGGTGCTTCA & CAAATGGACT & GAATGGCTCA & 840 \\
\hline AGAGCAAGAA & CGTGGGATTA & CTATTACATC & TGCTGCTACA & АCTGCATTCT & GAAAAAACAC & 900 \\
\hline AAGATTTAAC & ATCATTGATA & СTCCTGGTCA & CGTAGATTTC & ACTGTTGAAG & TTGAACGTTC & 960 \\
\hline ATTAAGAGTT & TTAGATGGTG & CTGTTGCTGT & TTTAGATGGT & CAATCTGGTG & TTGAACCTCA & 1020 \\
\hline AACTGAAACT & GTTTGAAGAC & AAGCAACAAA & TTATAAAGTT & CCTCGTATTG & TTTTTGTTAA & 1080 \\
\hline TAAAATGGAT & AAAACAGGTG & CTGATTTTAT & TTATTCAGTA & AAAACTATTG & GTGATAGATT & 1140 \\
\hline AGGTGCTAAA & GCTGCCCCAA & TTCAATTACC & AATTGGAGCT & GAAGAAAACT & TCACAGGTAT & 1200 \\
\hline TATTGATTTA & GTTGAAATGA & AAGCTTATGA & GTTTGATGGA & AAACCTGAAG & AAAATTATAA & 1260 \\
\hline AGAAATTGAA & АТTCCAACTA & ATTTATTAGA & ACAAGCTAAA & GAATTAAGAG & CACATTTAGT & 1320 \\
\hline TGAAGTTGCT & GTTGAATATG & ATGAAGAATT & ATTAATGAAA & TTTTTAGATG & GTGGAGAAAT & 1380 \\
\hline TAGTATTTCT & GAATTAAAAT & CAGCAATTAG & AAAAGGTGTA & ATTAATGCTG & АTTTCTTCCC & 1440 \\
\hline AGTATTAGCT & GGTTCAGCAT & TTAAAAATAA & AGGTGTTAAA & TTATTATTAG & ATGCTGTTGT & 1500 \\
\hline & & & & & & \\
\hline
\end{tabular}

FIG. 5. Nucleotide sequence of the insert in the recombinant M13 clone CAP-21. The start codons of the two possible coding regions are boxed, and the stop codon of the first is underlined.

digested with the restriction endonuclease $R s a$ I. The DNA fragments were transferred onto nitrocellulose as described previously. When hybridizations were done with the F38-12 probe, only $M$. capricolum and the F38 mycoplasma produced hybridization bands, and the other members of the cluster were not detected. The F38-12 probe produced a single hybridization band for both $M$. capricolum and the F38 mycoplasma. The bands were well separated and differentiated the two organisms (Fig. 4).

Sequence analysis. The CAP-21 insert was determined to be 1,525 bases in length (Fig. 5), and the base composition was $17.7 \% \mathrm{G}$ ( 271 bases), $38.9 \% \mathrm{~A}$ (596 bases), $31.5 \% \mathrm{~T}$ (475 bases), and $11.9 \% \mathrm{C}$ (183 bases). Plots of potential open reading frames in all six frames showed two possible open reading frames. The nucleotide sequence of the F38-12 insert was 464 bases in length (Fig. 6). It had a base composition of $12.7 \% \mathrm{G}$ (59 bases), 26.3\% A (122 bases), 43.5\% T (202 bases), and $17.5 \% \mathrm{C}$ (81 bases). Plots in all six frames did not show any open reading frames of significant length.

Specificity of probe. Experiments were done to determine the specificity of the two probes. TaqI digests of genomic DNA from the mycoplasmas $A$. laidlawii, $M$. orale, $M$. hyorhinis, and $M$. arginini and two representative bacteria, $P$. multocida and $E$. coli, together with TaqI digests of members of the $M$. mycoides cluster were tested by using CAP-21. Hybridization bands were produced with all of these organisms; however, signals that resulted from distantly related organisms were much weaker and had patterns distinctly different from those from mycoplasmas belonging to the cluster (results not shown).

When $R s a$ I digests of DNA from the same organisms were hybridized with the F38-12 probe, only F38, M. capricolum, and $E$. coli produced hybridization bands. The signal from $E$. coli was distinct in pattern from those of the F38 mycoplasma and $M$. capricolum (results not shown).

Sequence data. Regions homologous to the CAP-21 insert were amplified from representative strains of each member of the cluster and sequenced. The sequence data are shown in Fig. 7. There is a high degree of homology, at least $96 \%$, between all members of the cluster, with most of the differences being concentrated in the two regions between nucleotides 63 and 81 and nucleotides 613 and 630 . A phylogenic tree was constructed with these sequence data (Fig. 8). The dendogram showed that $M$. capricolum and the

$\begin{array}{lllllll}\text { GAATTCTAAT } & \text { AAAAAAAGAA } & \text { AATATTTTTT } & \text { GCTAAAAAAT } & \text { TATTCTCTTC } & \text { ACTAAAATCA } & 60 \\ \text { TATTTTTATT } & \text { AGAAATCTTA } & \text { AAAATTGTTT } & \text { TAATAAATTC } & \text { TAAATTAAAG } & \text { ATCTAAAGTA } & \mathbf{1 2 0} \\ \text { TGACTATCCC } & \text { ATCCAAAATC } & \text { TATCCCCAAT } & \text { GAACTATTAC } & \text { TTGATAAAGG } & \text { TGCTGCCATT } & 180 \\ \text { GGTTGATCAC } & \text { TTGGTTTTT } & \text { AAGTTCATTA } & \text { ACTTTTGCTT } & \text { TAGCAGCATT } & \text { TAATTCTTCT } & \mathbf{2 4 0} \\ \text { TCTGCTTTTT } & \text { TAATTGCTGC } & \text { ATTAGCTGCT } & \text { TCTTCTCGCT } & \text { TTTTTATCTT } & \text { TTTTAGTTT } & \mathbf{3 0 0} \\ \text { AGCGTCTTCT } & \text { AAAGATTTT } & \text { TAGCGTCTTC } & \text { TAAAGATTTT } & \text { TGTGCTTTAG } & \text { TAACTGCTTC } & 360 \\ \text { TTGAGCCTTT } & \text { TTTAATTCAT } & \text { CTTGCTTAGC } & \text { TTGATCGCTT } & \text { GGTTCGCCTG } & \text { ATTCTCGGTT } & \mathbf{4 2 0} \\ \text { GATCACCTTG } & \text { AGTTCCTTCG } & \text { CTTTCAGAAG } & \text { TTTCACTTGA } & \text { ATTC } & & 464\end{array}$

FIG. 6. Nucleotide sequence of the insert in the recombinant M13 clone F38-12. 
10

$20 \quad 30 \quad 40 \quad 50$

60

70

80

90

100

CAP TTCTCAAGGACGTTCACTATATGGAGTAAAAAGACC-AAAAGTTAAAAAA TAATAAATCAAAT-----TA-ATAAGTGT-GTATATGAA---TTAAAAAG YGO TTCTCAAGGACGTTCGCTATATGGAGTAAAAAGACCTAAAAGTTAAAAAA TAATAAATCAAAT------TAAATAAGTTT-GTATATGAA---TTAAAAAG GLA TTCTCAAGGACGTTCACTATATGGAGTAAAAAGACC-AAAAGTTAAAAAA TAATAAATCAAAT- - - - TA-ATAAGTGT-GTATATGAA---TTAAAAAG TTCACAAGGACGTTCATTATATGGAGTAAAAAGACCT-AAAGTTAAAAAA TAAGAAAATAAATAGTAATAAATAAACCGTGTATATCAA--ATTAAAAAG TTCACAAGGACGTTCATTATATGGAGTAAAAAGACCT-AAAGTTAAAAAA TAATAAAACAAATAATAAGTAAACCGT---GTATATCAA--ATTAAAAAG TTCACAAGGACGTTCATTATATGGAGTAAAAAAACCT-AAAGTTAAAAAA TAATAAAACAAATAATAATAAGTAAACCGTGTATATCTCAAATTAAAAAG

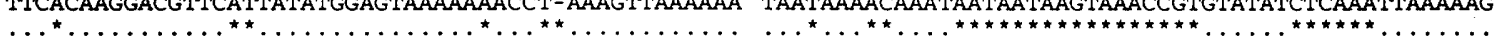

110

120

130

140

150

160

170

180

190

200

YGO

GLA

BG7

F38

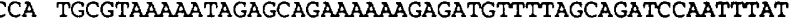
AACACATTAAAAGTAGCATATTTATATTAAAAAGAAAGGAGTTATTACCA TGCGTAAAAATAAAGCAGAAAAAAGAGATCTTTTAGCAGATCCAATTTAT AACACATTAA--GTAGCATATTTATATTAAAAAGAAAGGAGTTATTACCA TGCGTAAGAATAGAGCAGAAAAAAGAGATCTTTTAGCAGATCCAATTTAT AACACATTAA--GTAGCATATTTATATTAAAAAGAAAGGAGTTATTACCA TGCGTAAGAATAGAGCAGAAAAAAGAGATGTTTTAGCAGATCCAATTTAT AACACATTAA * " * . .

CAP

YGO

GLA

BG7

KID

F38

AATTCTAAATTAGTTACTCGTGCAATTAATAAAATTATGTTAGATGGTAA AAGAGGAATTGCTCAGTCAATTATTTATGATGCATTTAATATAATTAAAG AATTCTAAATTAGTTACTCGTGCAATTAATAAAATTATGTTAGATGGTAA AAGAGGAATTGCTCAGTCAATTATTTATGATGCATTTAATATAATTAAAG AATTCTAAATTAGTTACTCGTGCAATTAACAAAATTATGTTAGATGGTAA AAGAGGAATT-C---GTCAATTATTTATGATGCATTTAATATAATTAAAG AATTCTAAATTAGTTACTCGTGCAATTAATAAAATTATGTTAGATGGTAA AAGAGGAATTGCTCAGTCAATTATTTATGATGCATTTAATATAATTAAAG AATTCTAAATTAGTTACTCGTGCAATTAATAAAATTATGTTAGATGGTAA AAGAGGAATTGCTCAGTCAATTATTTATGATGCATTTAATATAATTAAAG AATTCTAAATTAGTTACTCGTGCAATTAATAAAATTATGTTAGATGGTAA AAGAGGAATTGCTCAGTCAATTATTTATGATGCATTTAATATAATTAAAG

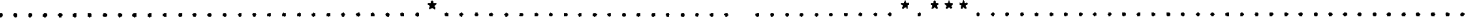

310

320

330

340

350

360

370

380

390

400 AAAAAACTAATAAAGAACCAATTGAAGTATTTAATAAAGCTATTGAAAAT ATTAAACCTCACTTAGAATTAAAAGTTCGTCGTATTGGAGGAGCTAACTA AAAAAACTAATAAAGAACCAATTGAAGTATTTAATAAAGCTATTGAAAAT ATTAAACCTCACTTAGAATTAAAAGTTCGTCGTATTGGAGGAGCTAACTA AAAAAACTAATAAAGAACCAATTGAAGTATTTAATAAAGCTATTGAAAAT ATTAAACCTCACTTAGAATTAAAAGTTCGTCCTATTGGAGGAGCTAACTA AAAAAACTAATAAAGAACCAATTGAAGTATTTAATAAAGCTATTGAAAAT ATTAAACCTCACTTAGAATTAAAAGTTCGTCGTATTGGAGGAGCTAACTA AAAAAACTAATAAAGAACCAATTGAAGTATTTAATAAAGCTATTGAAAAT ATTAAACCTCATTTAGAATTAAAAGTTCGTCGTATTGGAGGAGCTAACTA AAAAAACTAATAAAGAACCAATTGAAGTATTTAATAAAGCTATTGAAAAT ATTAAACCTCATTTAGAATTAAAAGTTCGTCGTATTGGAGGAGCTAACTA

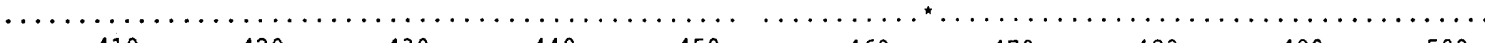
410 420 430 440 450 460 470 480 490 500

CAP TCAAGTTCCTGTAGAAGTTTCAGCTGAAAGACAAATCACTTTAGCTTTAC TCAAGTTCCTATAGAAGTTTAAGCTGAAAGACAAATCACTTTAGCTTTAC TCAAGTTCCTGTAGAAGTTTCAGCTGAAAGACAAATCACTTTAGCTTTAC TCAAGTTCCTGTAGAAGTTTCAGCTGAAAGACAAATCACTTTAGCTTTAC TCAAGTTCCTGTAGAAGTTTCAGCTGAAAGACAAATCACTTTAGCTTTAC GTTGATTAATTAATTATGCAAGATTAAGAAACGAAAAAGTTATGACAATT TTGATTAATTAATTATGCAAGATTAAGAAACGAAAAAGTTATGACAATT GTTGATTAATTAATTATGCAAGATTAAGAAACGAAAAAGTTATGACAATT GTTGATTAATTAATTATGCAAGATTAAGAAACGAAAAAGTTATGACAATT GTTGATTAATTAATTATCGAAGATTAAGAAACGAAAAAGTTATGACAATT GTTGATTAATTAATTATGCAAGATTAAGAAACGAAAAAGTTATGACAATT

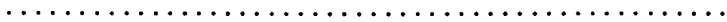
$\ldots \ldots \ldots \ldots \ldots \ldots$

510

520

530

$540 \quad 550$

560

570

580

590

600

YAP

YGO

AAATTAGCTAATGAAATTATTGATGCATCAAATAATATTGGTGGATCAGT AAATGAGCTAATGAAATTATTGATGCATCAAATAATATTGGTGGATCAGT AAATTAGCTAATGAAATTATTGATGCATCAAATAATATTGGTGGATCAGT AAATTAGCTAATGAAATTATTGATGCATCAAATAATTTTGGTGGATCAGT AAATTAGCTAATGAAATTATTGATCGATCAAATAATATTGGTGGATCAGT

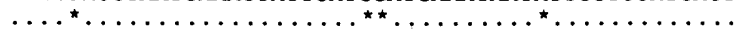

610

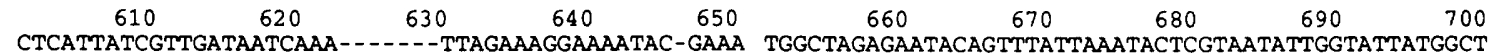

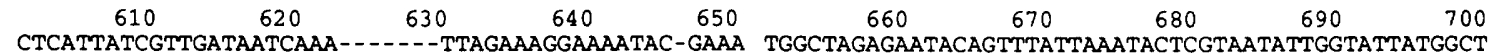

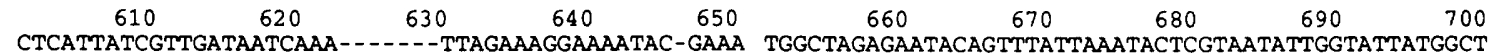

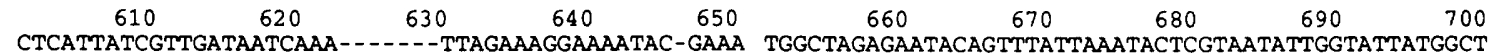
TAAAAAACGTGAAGATACTCATAAAATGGCAGAACGAAATAAAGCGTTTG TAAAAAACGTGAAGATACTCATAAAATGGCAGAAGCAAATAAAGCGTTTG TAAAAAaCGTGAaGATACTCATAAAATGGCAGAaGCAAATAAAGCATTTG

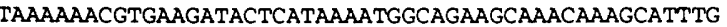
TAAAAAACGTGAAGATACTCATAAAATGGCAGAAGCAAACAAAGCATTTG YGO CTCATTATCGTTGATAATCAAA-.....--TTAGAAAGGAAAATAC-GAAA TGGCTAGAGAATACAGTTTATTAAATACTCGTAATATTGGTATTATGGCT GLA CACACTATCGTTGATAATCAAA------TTAGAAAGGAAAATAG-CAAA TGGCTAGAGAATACAGTTTATTAAATACTCGTAATATTGGTATTATGGCT

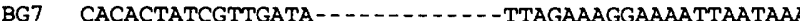
TGGCTAGAGAATACAGTTTATTAAATACTCGTAATATTGGTATTATGGCT CACACTATCGTTGAT---C--ATT-AGAATTAGAAAGGAAAATTAATAAA TGGCTAGAGAATACAGTTTATTAAATACTCGTAATATTGGTATTATGGCT CACACTATCGTTG--AAT - AATTAAGAATTAGAAAGGAAAATTAATAAA TGGCTAGAGAATACAGTTTATTAAATAATCGAAATATT-GAATTATGGCT

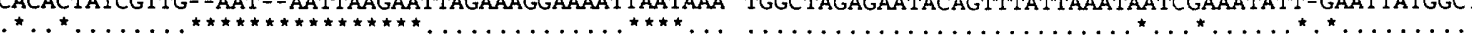

710 720 730 740 750 760 770 780 790 800 CAP CATATTGATGCTGGTAAGACTACTACTACTGAACGTATTTTATTTCATAC TGGAAAAATTCACAAAATAGGTGAACACATGAAGGTGCTTCACAAATGG

YGO CATATTGATGCTGGTAAGACTACTACTACTGAACGTATTTTATTTCATAC

GLA CATATTGATGCTGGTAAGACTACTACTACTGAACGTATTTTATTTCACAC

BG7 AATATTGATGCTGGTAAGACTACTACTACTGAACGTATTTTATTTCACAC TGGAAAAATTCACAAAATAGGTGAAACACATGAAGGTGCTTCACAAATGG TGGAAAAATTCACAAAATAGGTGAAACACATGAAGGTGCTTCACAAATGG TGGAAAAATTCATAAAATAGGTGAAACACATGAAGGTGCTTCACAAATGG AATATTGATGCTGGTAAGACTACTACTACTGAACGTATTTTATTTCACAC TGGAAAAATTCACAAAATAGGTGAAACACATGAAGGTGCTTCACAAATGG CATATTGATGCTGGTAAGACTACTACTACTGAACGTATTTTATTTCACAC TGGAAAAATTCACAAAATAGGTGAAACACATGAAGGTGCTTCACAAATGG

FIG. 7. Alignment of DNA sequences of regions corresponding to the CAP- 21 insert from representative members of the $M$. mycoides cluster; $M$. mycoides subsp. mycoides SC strain Gladysdale (GLA), M. mycoides subsp. mycoides LC strain Y Goat (YGO), $M$. mycoides subsp. capri ZZ (CAP), M. capricolum California Kid (KID), F38 mycoplasma original isolate (F38), and bovine group 7 mycoplasma strain R2222 (BG7). Differences (*) and similarities (.) in nucleotides are shown; dashes have been inserted in some sequences to aid alignment.

F38 mycoplasma are more closely related to each other than to the other members of the cluster, $M$. mycoides subsp. capri and $M$. mycoides subsp. mycoides LC are very closely related, and bovine group 7 mycoplasmas form a separate group.
Several changes were observed between the sequence of the CAP-21 insert and that derived from the genome of $M$. mycoides subsp. capri directly by PCR and asymmetric PCR. These changes were minor and were C-to-T transitions at nucleotides 1130,1403 , and 1406. 
810 ACTGAATGGCTCAAGAGCAAGAACGTGGGATTACTATTACATCTGCTGCT GLA ACTGAATGGCTCAAGAGCAAGAACGTGGGATTACTATTACATCTGCTGCT ACAACTGCATTCTGAAAAAACACAAGATTTAACATCATTGATACTCCTGG ACAACTGCATTCTGAAAAAACACAAGATTTAACATCATTGATACTCCTGG ACAACTGCATTCTGAAAAAATACAAGATTTAACATTATCGATACTCCAGG ACAACTGCATTCTGAAAAAACACAAGATTTAATATTATTGATACTCCAGG ACA-CTGCATTCTGAAAAAATAC-AGATTTAATATTATTGATACTCCAGG

920

930

940

950

960

970

980

990

1000

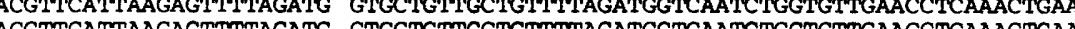

GLA TCACGTAGATTTCACTGTTGAAGTTGAACGTTCATTAAGAGTTTTAGATG

BG7 TCACGTAGATTTTACTGTTGAAGTTGAACGTTCATTAAGAGTTTTAGATG

KID TCACGTAGATTTTACTGTTGAAGTTGAACGTTCATTAAGAGTTTTAGATG

F38 TCACGTAGATTTTACTGTTGAAGTTGAACGTTCATTAAGAGTTTTAGATG GTACTGTTGCTGTTTTAGATGGTCAATCTGGTGTTGAGCCTCAAACTGAA TTCCTTTGCT GTGCTGTTGCTGTTTTAGATGGTCAATCTGGTGTTGAGCCTCAAACTGAA GTGCTGTTGCTGTTTTAGATGGTCAATCTGGTGTTGAGCCTCAAACTGAA ATTGGTGATAGATTAGGTGCTAAAGCTGCTCCAATTCAATTACCAATTGG ATTGGTGATAGATTAGGTGCTAAAGCTGCTCCAATTCAATTACCAATTGG AGCTAATA AGCTGAAGAAAACTTCACAGGTATTATTGATTTAGTTGAAATGAAAGCTT AGCTGAAGAAAACTTCACAGGTATTATTGATTTAGTTGAAATGAAAGCTT AGCTGAAGAAAACTTCACAGGTATTATTGATTTAGTTGAGATGAAAGCTT ATGAGTTTGACGGAAAACCTGAAGAAAATTATAAAGAAATAGAAATTCCA $\begin{array}{ccccr}1260 & 1270 & 1280 & 1290 & 1300 \\ \text { ACTAATTTATTAGAACAAGCTAAAGAATTAAGAGCACATTTAGTTGAGT }\end{array}$ ACTAATTTATTAGAACAAGCTAAAGAATTAAGAGCACATTTAGTTGAAGT ACTAATTTATTAGAACAACTAAGAATTAAGAGCACATTTAGTTGAAGT ACTAATTTATTAGAACAAGCTAAGGATTAAGAGCGCATTTAGTTGAAGT ACTAATTTATTAGAAC--GCTAAAGAAT-AAGAGCACATTTAGTTGAAGT $\begin{array}{lllll}1310 & 1320 & 1330 & 1340 & 1350\end{array}$ $\begin{array}{ll}\text { CAP } & \text { TGCTGTTGAATATGATGAAGAATTATTAATGAAATTTTTAGATGGTGGAG } \\ \text { YGO } & \text { TGCTGTTGAATATGATGAAGAATTATTAATGAAATTTTAGATGGTGGAG }\end{array}$

YGO TGCTGTTGAATATGATGAAGAATTATTAATGAAATTTTTAGATGGTGGAG
GLA TGCTGTTGAATATGATGAAGAATTATTAATGAAATTTTTAGATGGTGGAG

BG7 TGCTGTTGAATATGATGAAGAATTATTAATGAAATTTTTAGATGGTGGAG

KID TGCTGTTGAGTATGATGAAGAATTACTAATGAAATTTTAGATGGTGGAG

F38 TGCTGTTGAGTATGATGAAGAATTACTAATGAAATTTTAGATGGTGGAG

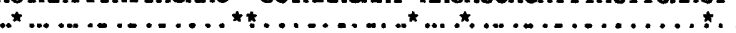
TGCTGTTGAGTATGATGAAGAATTACTAATGAAATTTTAGATGGTGGAG

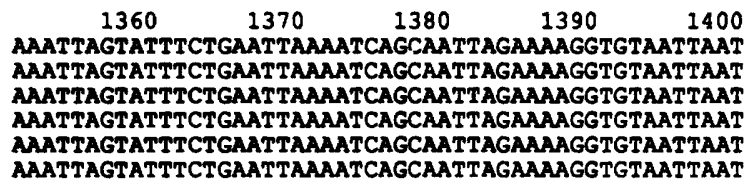

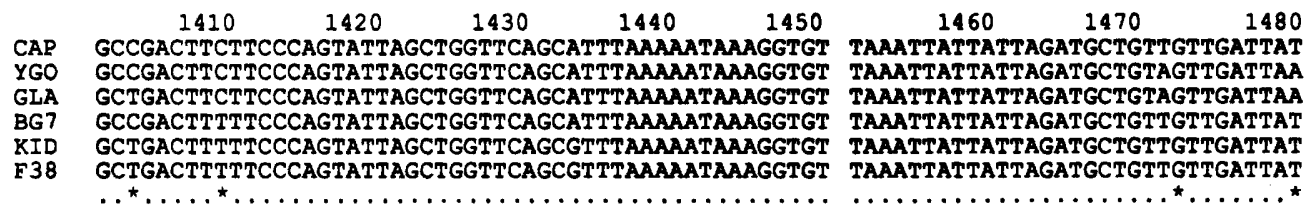

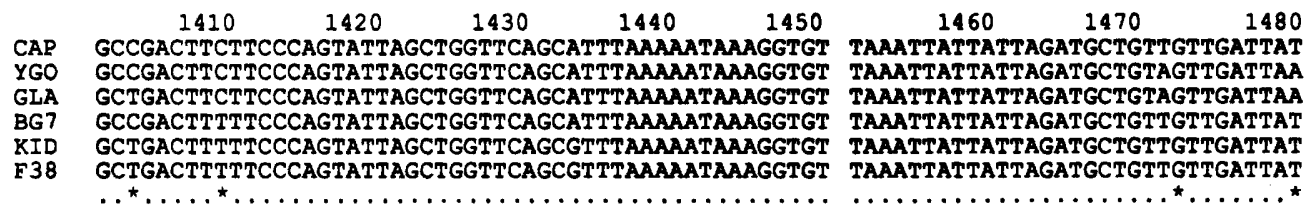

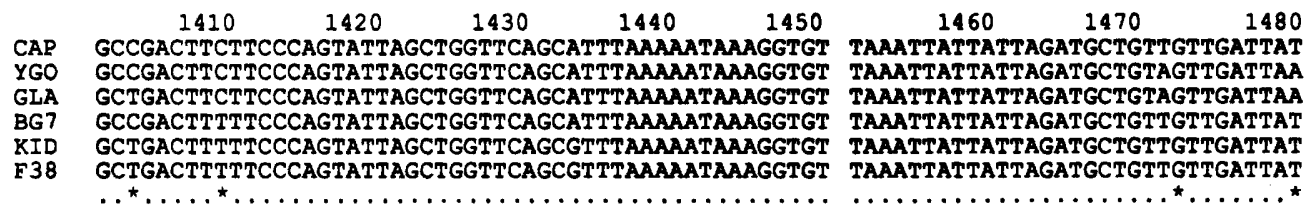

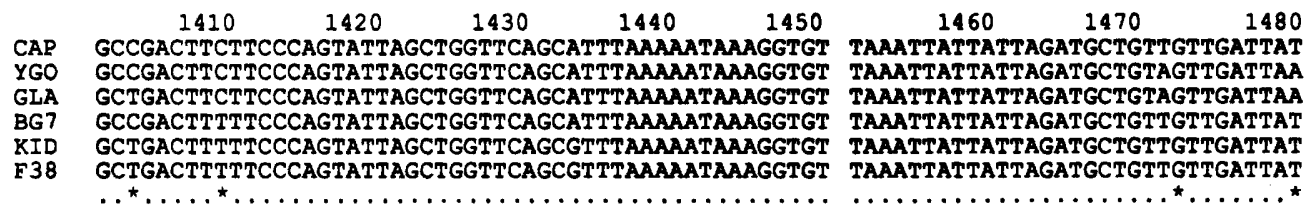

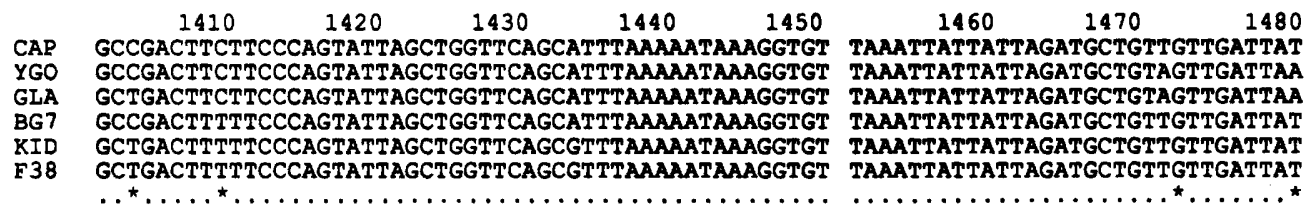
FIG. 7-Continued.

\section{DISCUSSION}

The organisms assigned to the $M$. mycoides cluster have been shown to be related to each other by a variety of tests, but classification of individual members within the cluster has proved to be difficult. We have studied the genetic relationships between the members of the cluster by using a DNA probe (CAP-21) which was originally prepared for the differentiation of the two types of $M$. mycoides subsp. mycoides. This probe was useful as a definitive test for the differentiation of strains of this organism capable of causing CBPP from those which did not produce the disease (19). When genomic DNA from each member of the $M$. mycoides cluster was digested with the restriction endonuclease TaqI and probed with CAP-21, a pattern of hybridization which indicated that this probe could be useful in the classification of the members of the $M$. mycoides cluster resulted.

The classification of $M$. mycoides subsp. mycoides LC within the cluster has been a difficult problem. DNA-DNA hybridization studies estimated that DNA of the type strain of $M$. mycoides subsp. mycoides SC, PG1, had a $90 \%$ homology with DNA from $M$. mycoides subsp. mycoides LC strain Y Goat (2). When PG1 was compared with the type strain of $M$. mycoides subsp. capri, PG3, $80 \%$ homology was found. These figures indicated Y Goat was more closely related to PG1 than was PG3. Several workers using 2D PAGE of acidic cellular proteins concluded the opposite (15, 16). TaqI-restricted DNA from Y Goat, when probed with CAP-21, produced a single band; PG3 produced a band in a 


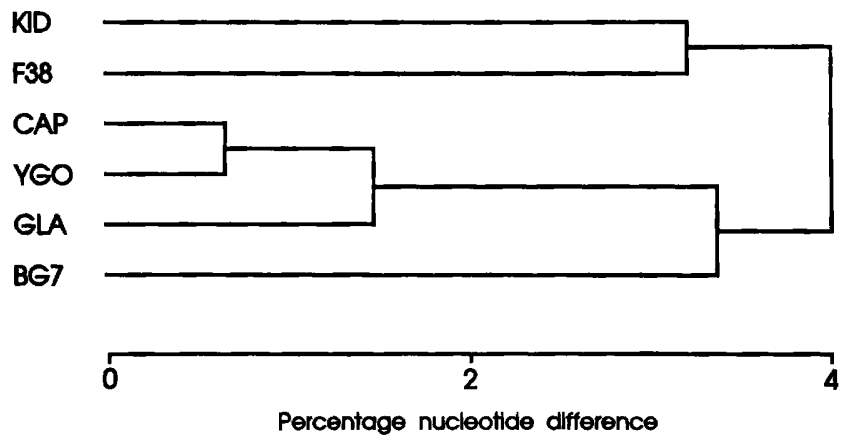

FIG. 8. Phylogenic tree constructed from comparisons of sequence data coding for regions corresponding to the CAP-21 probe in each member of the $M$. mycoides cluster. CAP, $M$. mycoides subsp. capri; GLA, $M$. mycoides subsp. mycoides SC; YGO, $M$. mycoides subsp. mycoides LC; KID, $M$. capricolum; F38, F38 mycoplasma; BG7, bovine group 7 mycoplasma.

similar position. PG1 also produced a single band but in a position different from those produced by Y Goat and PG3. This shows that Y Goat and PG3 are more closely related to each other than to PG1, and these results agree with those of the 2D-PAGE studies. We have compared eight strains of $M$. mycoides subsp. mycoides SC and found them to be a very homogeneous group (19). Further evidence of this differentiation has been shown by Costas and associates (6), who, using 1D PAGE, found that strains of $M$. mycoides subsp. mycoides SC made up a distinct phenon quite separate from a phenon comprising both $M$. mycoides subsp. mycoides $\mathbf{L C}$ and $M$. mycoides subsp. capri.

We have compared nine strains of $M$. mycoides subsp. mycoides LC and five strains of $M$. mycoides subsp. capri (Fig. 2). Some variability in mobility of the hybridizing band is seen, indicating generally minor variations in the genomes of these organisms. Most (i.e., 11) of the 14 strains are similar to M. mycoides subsp. capri PG3 and may belong to a common group. However, three of the strains tested (N108, EZG, and F30) appear to be different. It is likely that strains N108 and EZG are the same organism, although serologically N108 has been previously identified as $M$. mycoides subsp. capri while EZG was identified as $M$. mycoides subsp. mycoides LC. A result similar to this was reported by Leach and associates (13), who found that strain N108 was one of two strains that fell outside a large phenon that comprised all the $M$. mycoides subsp. mycoides LC and $M$. mycoides subsp. capri strains tested. The F30 strain appears to be unrelated to either the $M$. mycoides subsp. mycoides LC or M. mycoides subsp. capri group. All three of these variant strains share some homology, as evidenced by the similar positions of one of the bands of hybridization.

The bovine group 7 mycoplasmas have remained an unclassified group within the $M$. mycoides cluster. Hybridization studies of this group using CAP-21 clearly separated them from all the other members of the $M$. mycoides cluster. This separation has previously been proposed on the basis of isoenzyme studies (17). Costas and colleagues (6), by $1 \mathrm{D}$ PAGE, also found that this group formed a phenon separate from all the other members of the $M$. mycoides cluster at a 75\% similarity level. Christiansen and Ernø (5), using DNA homology studies, found that bovine group 7 mycoplasmas were equally related to $M$. mycoides and $M$. capricolum with homologies of $60 \%$ and on this basis should be recognized as a separate species.
The CAP-21 probe was unable to distinguish between $M$. capricolum and the F38 mycoplasma, indicating that the organisms are very similar. Further evidence that these two mycoplasmas have a close relationship has been shown serologically $(3,4)$; also, in DNA homology studies they showed an $80 \%$ relatedness (5). Rodwell (16) and Andersen and coworkers (1) found a $42 \%$ protein congruence for F38 and $M$. capricolum which was consistent with the $80 \%$ DNA homology value from reference 5. CCPP, a disease of economic importance in Africa, is caused by the F38 mycoplasma, and there is a need to have a reliable method for its diagnosis and to monitor its spread. Problems in the identification of the organism have been compounded by serological cross-reactions with other members of the cluster. The F38 mycoplasma produces very strong cross-reactions with the bovine group 7 mycoplasma $(4,11)$. F38 field strains that cross-react with $M$. capricolum have also been isolated (4). Confusing variations in biochemical characteristics of an isolate which was identified as $M$. capricolum but serologically resembled F38 mycoplasma were also reported (4). A second probe which could specifically differentiate between these two organisms was developed. With these two probes is it possible not only to classify all the members of the $M$. mycoides cluster but also for a diagnostic laboratory to identify specifically the agents which cause CBPP and CCPP.

Comparison of nucleotide sequences of each member of the $M$. mycoides cluster over the region delineated by the CAP-21 probe enabled the construction of a phylogenic tree which demonstrated relationships between them. The results are similar to those obtained when the cluster was studied by hybridization analysis of whole cellular DNA. The dendrogram showed a maximum variation between members of $4 \%$, with $M$. mycoides subsp. mycoides SC and LC and $M$. mycoides subsp. capri being closely related and with $M$. mycoides subsp. mycoides $\mathrm{LC}$ being more closely related to $M$. mycoides subsp. capri than to $M$. mycoides subsp. mycoides SC. $M$. capricolum and the F38 mycoplasmas were also grouped with a closer relationship to each other than to the other members of the cluster. Bovine group 7 mycoplasmas are most closely related to $M$. mycoides subsp. mycoides SC but are more distantly related to the other members of the cluster.

\section{ACKNOWLEDGMENTS}

We thank K. Sproat and T. Chamberlain for excellent technical assistance.

\section{REFERENCES}

1. Andersen, H., G. Christiansen, and C. Christiansen. 1984. Electrophoretic analysis of proteins from Mycoplasma capricolum and related serotypes using extracts from intact cells and from minicells containing cloned mycoplasma DNA. J. Gen. Microbiol. 130:1409-1418.

2. Askaa, G., H. Ernø, and M. O. Ojo. 1978. Bovine mycoplasmas: classification of groups related to Mycoplasma mycoides. Acta Vet. Scand. 19:166-178.

3. Bolske, G., H. Msami, N. E. Humlesjo, H. Ernø, and L. Jonsson. 1988. Mycoplasma capricolum in an outbreak of polyarthritis and pneumonia in goats. Acta Vet. Scand. 29:331-338.

4. Chingi, A. K., H. Ernø, and P. G. Waiyaki. 1987. Report on serological problems in classification of mycoplasmas from cases of contagious caprine pleuropneumonia in Kenya, p. 85-89. In G. E. Jones (ed.), Agriculture: contagious agalactia and other mycoplasmal diseases of small ruminants. Office for Official Publications on the European Communities, Luxembourg, Luxembourg. 
5. Christiansen, C., and H. Ernø. 1982. Classification of the F38 group of caprine mycoplasma strains by DNA hybridization. J. Gen. Microbiol. 128:2523-2526.

6. Costas, M., R. H. Leach, and D. L. Mitchelmore. 1987. Numerical analysis of PAGE protein patterns and the taxonomic relationships within the 'Mycoplasma mycoides cluster.' J. Gen. Microbiol. 133:3319-3329.

7. Cottew, G. S. 1979. Caprine-ovine mycoplasmas, p. 103-132. In J. G. Tully and R. F. Whitcomb (ed.), The mycoplasmas, vol. 2. Academic Press, Inc., New York.

8. Cottew, G. S., A. Breard, A. J. DaMassa, H. Ernø, R. H. Leach, P. C. Lefevre, A. W. Rodwell, and G. R. Smith. 1987. Taxonomy of the Mycoplasma mycoides cluster. Isr. J. Med. Sci. 23:632 635.

9. Cottew, G. S., and F. R. Yeats. 1978. Subdivision of Mycoplasma mycoides subsp. mycoides from cattle and goats into two types. Aust. Vet. J. 54:293-296.

10. Felsenstein, J. 1989. PHYLIP 3.2 manual. University of California Herbarium, Berkeley.

11. Kanyi Kibe, M., D. E. Bidwell, P. Turp, and G. R. Smith. 1985. Demonstration of cross-reactive antigens in F38 and related mycoplasmas by enzyme-linked immunosorbent assay (ELISA) and immunoblotting. J. Hyg. Camb. 95:95-106.

12. Knowles, N. J. 1990 . A method for direct nucleotide sequencing of foot-and-mouth disease virus RNA for epidemiological studies, appendix 06-112. In Report of the session of the Research Group of the Standing Technical Committee of the European Commission for the Control of Foot-and-Mouth Disease, Lindholm, Denmark, 25 to 29 June, 1990. FAO, Rome.
13. Leach, R. H., M. Costas, and D. L. Mitchelmore. 1989. Relationship between Mycoplasma mycoides subsp. mycoides ('large-colony' strains) and $M$. mycoides subsp. capri, as indicated by numerical analysis of one-dimensional SDS-PAGE protein patterns. J. Gen. Microbiol. 135:2993-3000.

14. McCabe, P. C. 1990 . Production of single-stranded DNA by asymmetric PCR, p. 76-83. In M. A. Innis, D. H. Gelfand, J. J. Sninsky, and T. J. White (ed.), PCR protocols: a guide to methods and applications. Academic Press, San Diego, Calif.

15. Nascimento, E. R., M. da G. F. Nascimento, E. A. Freundt, and H. Andersen. 1986. Isolation of Mycoplasma mycoides from outbreaks of caprine mycoplasmosis in Brazil. Br. Vet. J. 142:246-257.

16. Rodwell, A. W. 1982. The protein fingerprints of Mycoplasmas. Rev. Infect. Dis. 4(Suppl. May-June):S8-S17.

17. Salih, M. M., H. Ernø, and V. Simonsen. 1983. Electrophoretic analysis of isoenzymes of mycoplasma species. Acta Vet. Scand. 24:14-33.

18. Smith, G. R. 1983. Examination of the subspecies and types of $M$. mycoides by mycoplasmaemia and cross-immunisation tests in mice, p. 47-53. In S. A. Hall (ed.), Agriculture: the diagnosis of contagious bovine pleuropneumonia and other infections with Mycoplasma mycoides subspecies mycoides. Office for Official Publications on the European Communities, Luxembourg, Luxembourg.

19. Taylor, T. K., J. B. Bashiruddin, and A. R. Gould. Application of a diagnostic DNA probe for the differentiation of the two types of Mycoplasma mycoides subspecies mycoides. Res. Vet. Sci., in press. 